\title{
La representación cartográfica del mundo en la Edad Media
}

\author{
tomás Franco Aliaga \\ JuLIO López-DavaliLlo LARREA \\ Departamento de Geografía. UNED
}

\begin{abstract}
RESUMEN
El Cristianismo y el Islamismo son dos paradigmas no sólo en el orden religioso y moral sino también en el científico, es decir, en la interpretación del origen del mundo y consecuentemente en la representación del mismo. Ya no se guía el hombre por la razón y la experiencia, por ejemplo, para explicar los movimientos de la tierra. Como creyente se ve obligado a seguir las pautas que le marcan los textos sagrados. La investigación deja paso a la fantasia y la cartografía se transforma en un mosaico de simbolos no siempre fáciles de interpretar. El Cristianismo rompe con la tradición clásica y vive una primera fase de absoluta simplificación cartográfica, como se comprueba en los mapamundis en O-T y en los Beatos. En el Islamismo, por el contrario, se deja sentir desde el primer momento la influencia helenistica, cuyos pueblos y bibliotecas han caido bajo el dominio de aquél.
\end{abstract}

\section{PALABRAS CLAVE}

Cristianismo, simbologia, interpretación religiosa, mapamundis $O-T$, Beatos, Portulanos, Islamismo, traducción, libros de viajes.
SUMMARY

Christianity and Islamism are two paradigms not only at a religious and moral level but also at a scientific one. That is, concerning the origins of the world and subsequently its representation. Man no longer explains the Earth movements based on reason and experience, but as a believer he feels drawn to following the guidance indicated by the holy texts. Investigation gives way to fantasy and cartography changes into a wealth of symbols not always easy to interpret. Christianity breaks off with classical traditions and begins a phase of absolute cartographic simplification, as one can see on the world maps in O-T and in the Beatos. Islamism, on the other hand, showed a Greek influence from the beginning, whose peoples and libraries have now fallen under the domain of the former.

\section{KEY WORDS}

Christianity, symbols, reiigious interpretation, world maps in O-T, Beatos, Portulanos, Islamism, translation, travel guides. 


\section{INTRODUCCIÓN}

El marco geográfico en el que se mueven los siglos medievales, ya sea entre los pueblos cristianos o del Islam, se alimenta de la tradición grecolatina, aunque muy mediatizada por dos elementos característicos: la religiosidad y la fantasia narrativa. Hay, sin embargo, algunas diferencias notables entre los geógrafos de uno y otro credo. Los cristianos han cortado casi todos los vínculos con la tradición geográfica a través de sus textos; los musulmanes en cambio ocupan parte de los territorios helenísticos y están en permanente relación con una bibliografía clásica que conocen y traducen a su idioma.

\section{LA GEOGRAFIA RELIGIOSA Y FANTÁSTICA CRISTIANA}

La hegemonia del Cristianismo supuso el rechazo de gran parte del legado científico griego, pero sobre todo de su espíritu crítico y de su laicismo. Todo el patrimonio científico cristiano o estaba contenido en los textos sagrados o no podía ir en contra de sus enseñanzas. Así lo expresa con contundencia el apologista Lucio C. Lactancio, del siglo III (1990), para quien la ciencia es en si misma ridícula y falsa, opinión explicable desde los fervores suscitados por la tolerancia al cristianismo en el Edicto de Milán (313), promulgado por Constantino el Grande.

Los planteamientos dogmáticos del Cristianismo comulgan mal con la postura racionalista grecolatina. De ahi el abandono, cuando no el rechazo y la beligerancia, de toda la ideología pagana. Las propuestas geográficas sobre el origen, composición y movimientos de la tierra sufren idéntica revisión, como no podía ser de otra manera. Casi nada de lo antiguo es válido y ha de pasar por el cedazo de las Escrituras Sagradas y de la fe. Es bien sabido que todo nuevo paradigma epistemológico barre por completo al anterior: ley de vida para unos, los vencedores, y de muerte para otros, los perdedores. En este caso el enfrentamiento surge entre una concepción revelada y celestial en auge y otra terrenal que se guía por la luz de la razón.

Se pierden numerosos conocimientos de la cultura clásica, pero sobre todo se abandona la curiosidad por el saber contrastado desde la razón. Osorio, Beda el Venerable y San Isidoro de Sevilla son las estrellas polares para Occidente. En las Etimologías de este último autor apunta más la influencia de Plinio el Viejo que la de los pensadores griegos, y eso que el escritor romano ya es de por sí una mala copia de éstos.

El proceso de degradación hace de los conocimientos geográficos occidentales una caricatura, cuando no una bufonada, de lo que fueron durante el esplendor helénico. La cosmografía se torna en cosmología (Ortega, V., 2000) y la representación de la tierra (mapamundi) en un ideograma, en un amasijo de símbolos no siempre fáciles de interpretar. Fruto de esta concepción simplista son los mapa- 
mundis en O-T y los Beatos, aunque éstos últimos proporcionan algo más de información que los otros.

En la obra isidoriana aparece representado el mundo conocido en forma de $\mathrm{O}$ (orbis) en cuyo interior se distribuyen los tres continentes, configurando una $\mathrm{T}$ (terrarum): Europa a la izquierda, África a la derecha y Asia arriba, cuna del Paraiso Terrenal. Este es el primer mapa que se conserva salido de la imprenta en 1472, ilustrando las Etimologías de San Isidoro, como si fuera un homenaje a todo un largo y oscuro pasado de la ciencia geográfica (fig. 1).

Procede de una versión impresa de la obra Etymologiarum sive originum libri XX en la ciudad alemana de Augsburg (1472). Es una representación simbólica de la Tierra, más vinculada con la cosmogonía bíblica que con la geografía científica. El jeroglífico de San Isidoro consta de una $O$ que encierra el mundo y de una T que separa los continentes conocidos: Europa, Asia y África. Un brazo de ese océano que envuelve la Tierra es el mar Mediterráneo, que ocupa el trazo vertical y horizontal de la T. Hay numerosas variantes sobre la misma base descrita. En la mayoría de los casos Jerusalén ocupa el centro del dibujo como una fijación cartográfico-religiosa (Franco, 2001).

Los mapas del Beato de Liébana (siglo VIII, Cantabria) son una mezcla de enclaves geográficos y de simbolismo religioso que, como miniaturas, decoran su libro Comentarios sobre el Apocalipsis (fig. 2). Otros autores cristianos, que no cartógrafos, siguen enriqueciendo los mapas mentales con ensoñaciones religiosas, referencias literarias y noticias verídicas de viajeros y mercaderes. Todo vale si engalana, fortalece y acrecienta la fe.

El Beato de la figura 2 es un manuscrito en color sobre pergamino $(36,5 \times 56,4$ $\mathrm{cm}$ ) que representa los tres continentes. Forma parte del libro Comentarios al Apocalipsis y al Libro de Daniel. Está orlado con peces y barcos y aparecen los nombres de los cuatro puntos cardinales con el cambio de orientación hacia el este. De ahí que occidens esté al Sur y septentrio al Oeste, por ejemplo. En el mapa serpentean importantes cursos de agua, se extienden varias cadenas montañosas simbolizadas como dientes de sierra y el Mediterráneo ocupa la zona centro, en sentido vertical, con Roma al oeste y Judea al este compartiendo protagonismo. En la parte superior, debajo de oriens, destaca el Paraiso Terrenal con Adán y Eva en el instante supremo de arriesgarlo por una manzana. Al este, y por influencia bíblica, cobra inusitada importancia el Mar Rojo (mare rubrum) con dos cuernos o golfos. En el mayor puede leerse con esfuerzo sinus arabicus y en el pequeño podemos intuir sinus indicus, aunque sin asegurarlo, lupa mediante. Como queda evidente, toda la toponimia está en latín.

En esta primera etapa medieval Occidente está encerrado en sí mismo, carece de la literatura fantástica y viajera del mundo islámico y no tiene, como sí ocurre con este último, una comunicación directa con la herencia escrita griega. Por otra parte, los viajes de los puebios nórdicos por las costas occidentales atlánticas no entran a formar parte del acervo cristiano hasta el siglo XVI. 


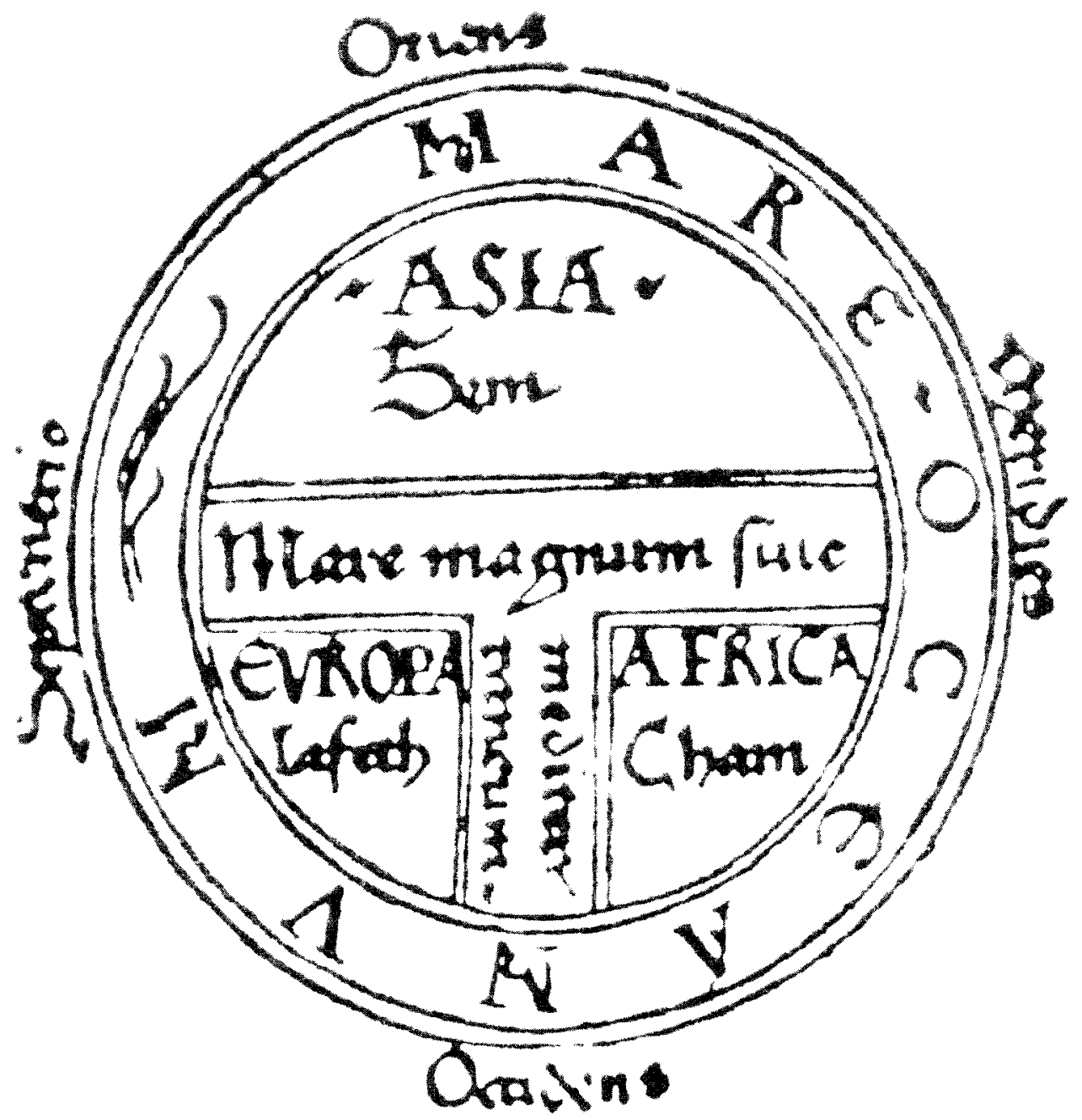

Fig. 1. Mapamundi Isidoriano Orbis Terrarum. Fuente: IGN. 1991.

Con el paso del tiempo empezaron a florecer la transcripción y el estudio de manuscritos en los monasterios, donde se refugió el saber occidental durante siglos, aunque siempre tutelado por la concepción biblica del universo, basada en estos cuatro principios: 1) Dios es el creador de los cielos y la tierra, según el Génesis. 2) La Humanidad también es creada a su imagen y semejanza después de haber colocado sobre la tierra a todos los demás seres. 3) La Naturaleza está al servicio del Hombre, como lo demuestra el hecho de que el Creador le instara a que pusiese nombre a todos los animales. 4) El pecado por desobediencia rompe el equilibrio entre la Naturaleza y el Hombre. 


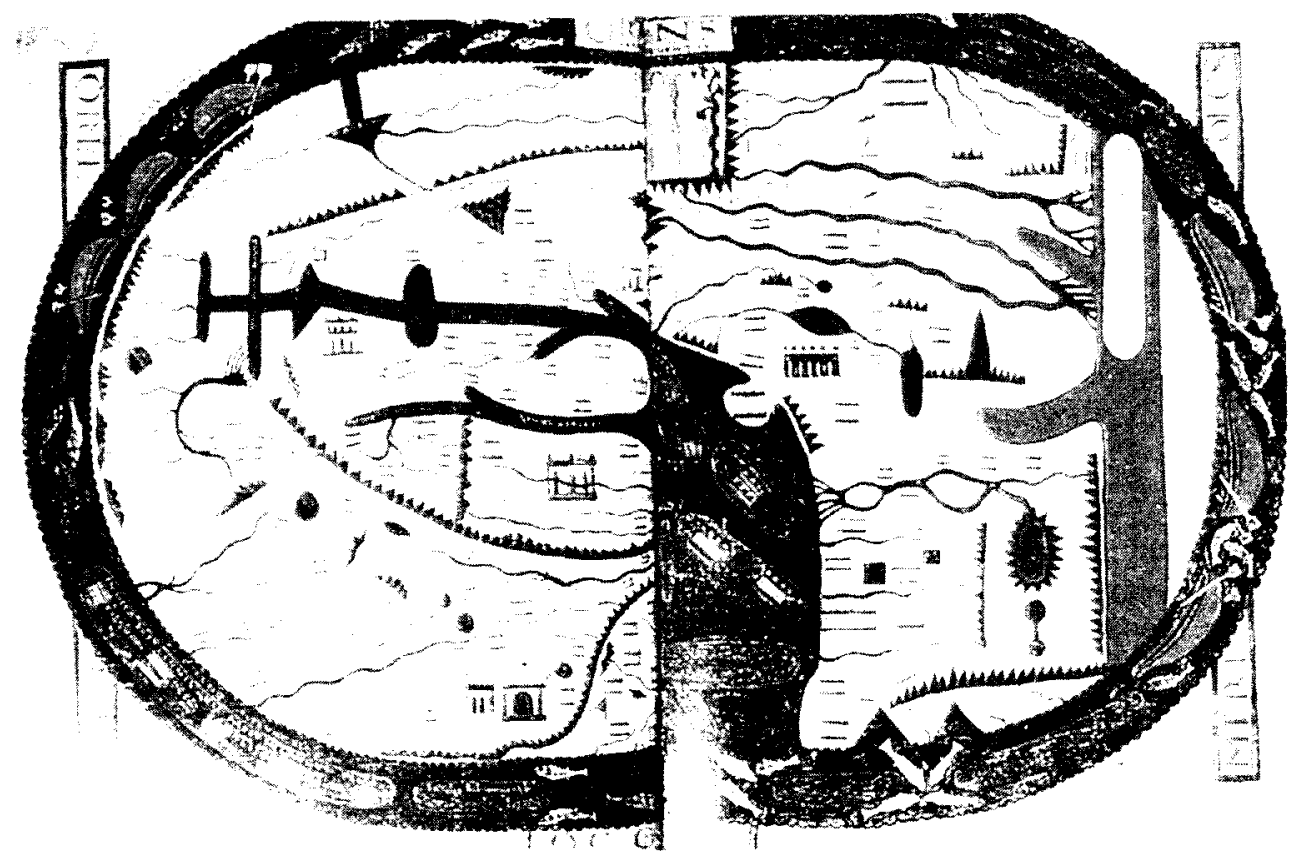

Fig. 2. Beato de Liébana (siglo XI). Fuente: Fundación de Santillana, 1992

Sabemos lo determinante que es la ideología en la evolución de la ciencia, a la que hay que añadir los intereses del poder y de la economía imperantes. Nadie puede dudar de que estos principios han determinado el desarrollo de la Geografía durante los siglos medievales en los que la visión teocrática del mundo está asumida por la sociedad en pleno.

Ya en ei siglo XIt empieza a despertarse el conocimiento geográfico a partir de las traducciones de textos árabes. Pero nunca aparecen las noticias geográficas como obras individualizadas sino dentro de publicaciones generales que recogen todos los saberes conocidos desde la medicina a la botánica pasando por las disquisiciones filosóficas, los viajes y la teología. En torno a tales parámetros enciclopédicos se mueve, por ejemplo, la General Historia de Alfonso X el Sabio (1221-1284).

La hegemonía del pensamiento religioso se va cuarteando poco a poco ante el auge de la navegación, cuyas necesidades diarias obligan al estudio de la cosmografía, a la elaboración de mapas ajustados a las entalladuras costeras y al invento de aparatos cada vez más precisos. Aparecen los portulanos, que son cartas de marear que reproducen con extraordinaria precisión los contornos de las costas mediterráneas, valiéndose de una rosa de los vientos más precisa (con 32 rumbos) y del uso de la escala y de la brújula.

Los rumbos, dibujados con largas y finas líneas, acaban formando una espesa urdimbre que enlaza con una abundante toponimia, escrita en latin o en catalán. 


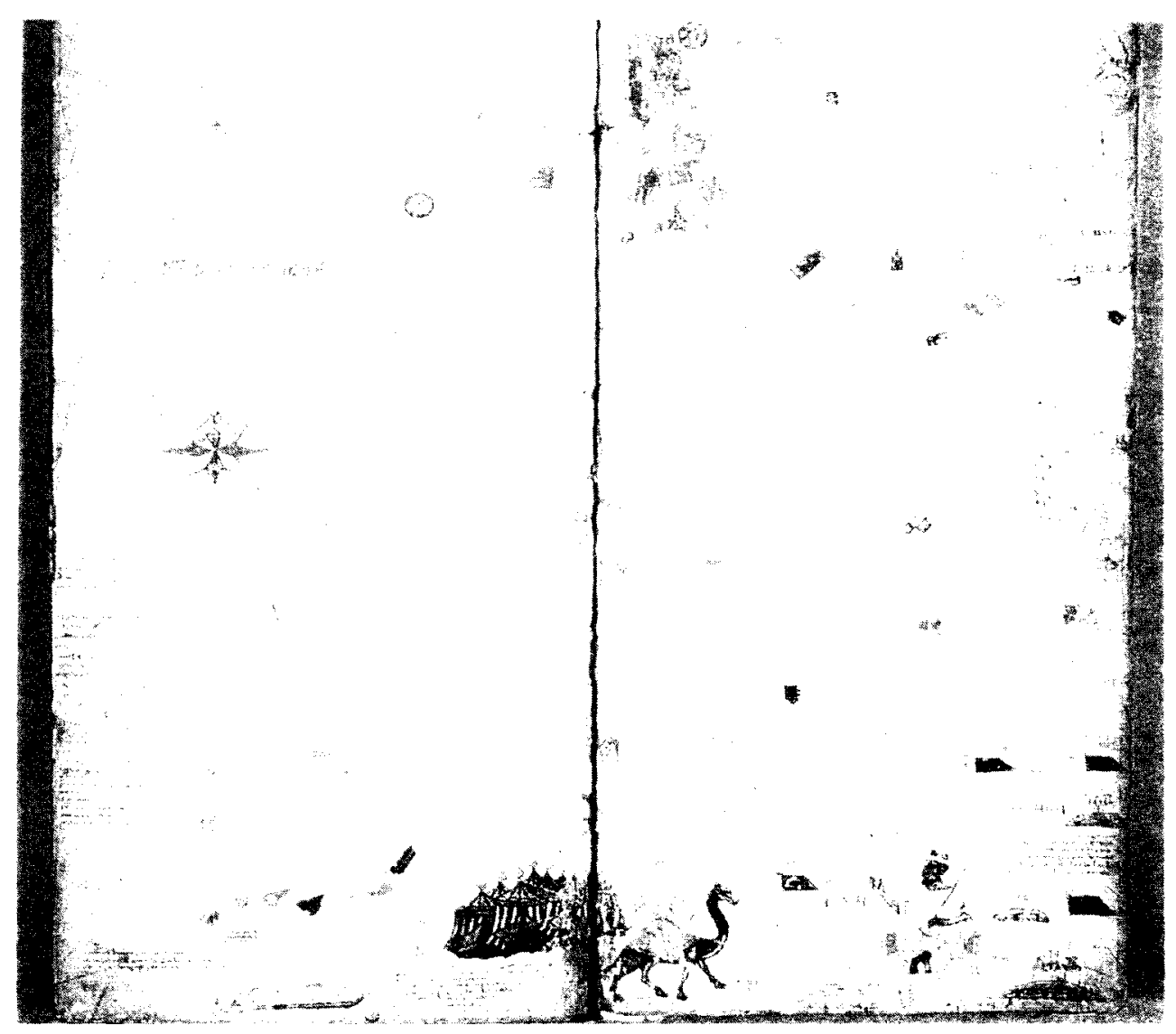

Fig. 3. Atlas catalán de 1.375 atribuido a Abraham Crespes (Biblioteca Nacional de Paris). Fuente: IGN. 1991

Los portulanos tuvieron su apoyo en los siglos xiv y xV, prolongándose hasta el XVII y cabe situarlos a caballo entre la cartografía y la obra de arte por la elegancia de los trazos y la profusión de adornos pictóricos que contienen (fig. 3).

Este atlas está considerado como un prototipo de la cartografía náutica y la obra cumbre de la escuela mallorquina, cuyos antecedentes hay que rastrearlos en la obra científica de Ramón Lull. Se compone de varias hojas de pergamino (piel de cordero), aunque en la figura sólo aparecen dos. La toponimia está en catalán y las leyendas o textos son explicaciones de utilidad para los comerciantes. Abundan las banderas para identificar los países; animales y objetos característicos de las regiones; ciudades amuralladas; cordilleras como los Alpes, Pirineos y el Gran Atlas y rios diversos entre los que llama la atención el Tajo en forma de báculo rodeando Toledo. El ámbito mediterráneo se representa con una gran exactitud, pero se mantiene un error que corregirá la cartografía moderna: el eje del Mediterráneo aparece distorsionado con un giro brusco hacia el noroeste. 


\section{CONTACTO DEL ISLAM CON LA TRADICIÓN GEOGRÁFICA CLÁSICA}

El primer impacto del Islam es catastrófico para la herencia clásica porque se pierden cientos de miles de manuscritos al incendiarse numerosas bibliotecas, pero en breve inician su meticulosa y eficaz labor de traductores. El mismo Occidente cristiano no tarda en beneficiarse de tal esfuerzo, pues hasta él van liegando no sólo los textos griegos traducidos sino también noticias sobre pueblos y culturas orientales e inventos varios como la brújula traída de China.

La peregrinación a La Meca representa para los árabes el mismo papel que el Camino de Santiago entre los cristianos. La atracción hacia los Santos Lugares fomenta el intercambio cultural y la elaboración de guias de viaje que describen pueblos, caminos, costumbres y regiones del trayecto. La mayoría son relatos destinados a maravillar y sorprender al lector. Hoy interesan por lo que sugieren sobre gustos, inquietudes o concepción del espacio y de los fenómenos, primero desde el centro cultural de Bagdag en el siglo Ix y más tarde desde A!-Andalus. Al igual que en el bando cristiano, ofrecen una visión del mundo fantástica, simple y sesgada, con La Meca como eje. Pero nada de todo esto llega hasta Europa.

Al-Idrisi (1099-1180) es el primero que influye realmente en el ámbito cristiano debido a sus relaciones con la corte de Roger II de Sicilia (1095-1154), para quien escribe el Libro de Rogerio o Recreo de quien desea recorrer el mundo. Desde aquí le resulta fácil obtener información sobre Europa y cumplir el encargo de hacer una descripción minuciosa acerca de las ciudades, gentes, tipos de agricultura, comercio, artesanía y creencias de quienes habitan en el interior de los siete climas en que divide el mundo (Jaubert, 1975).

Utiliza la información de Ptolomeo y la tradición griega para la descripción y representación de la tierra, y en su redacción invierte 15 años de continuos viajes. No faltan relatos fantásticos y noticias traídas de tiempos remotos a lo largo de sus páginas donde también incluye 70 mapas entre los que aparece la Península Ibérica con un gran lujo de detalles (fig. 4).

El tangerino Ibn Battuta (1304-1378) y su contemporáneo el tunecino Ibn Khaldun (1332-1406) son dos historiadores-geógrafos que recorren gran parte de las tierras orientales conocidas. El segundo tiene una concepción organicista de los procesos de la civilización y se mueve en el área de influencia ptolomeica, cuyo compendio de astronomia habia sido traducido con el nombre de Almagesto. Hace suya la descripción climática de Al-Idrisi y asegura que la mayor densidad de población está en las zonas centrales por sus condiciones más benignas. Da tanta importancia al medio climático que hace depender del mismo el carácter de sus habitantes (Ibn Khaldun, 1967), ideas que tuvieron su eco en el determinismo ambiental europeo del siglo xIx. (Unwin, 1995).

La Tabula Rogeriana de al Idrisi forma parte de una obra encargada por Roger II de Sicilia (1154). Como en la figura 2, también aquí está cambiada la orientación con el sur arriba, por lo que resulta obligado darle la vuelta al mapa para reconocerlo. Este mapa es el resultado de una numerosa red de informadores enviados a 
gran parte de los lugares conocidos de entonces. El resultado fue una cartografia bastante ajustada a la realidad, en la que no faltan siete franjas longitudinales para separar otros tantos climas, sin contar con las referencias a una zona tropical y otra ártica. En la obra se dibuja Al-Andalus o España con forma de triángulo distorsionado, pero perfectamente reconocible. Contiene notables aciertos como su situación entre los paralelos $44^{\circ}$ y $35^{\circ}$ (el correcto es $36^{\circ}$ ), orientación de las principales cadenas montañosas y cuencas fluviales. Supuso un enorme avance frente a la cartografía simbólica cristiana de la época y posterior, aunque aún contenga algunas referencias fantásticas como la forma de media luna que tiene el conjunto de África (inapreciable en este fragmento) y los montes de la luna, representados por ese barco, al sur de África, con tres lagos que alimentan el Nilo. Los trazos alargados y más gruesos repartidos por todo el mapa simulan cadenas montañosas.

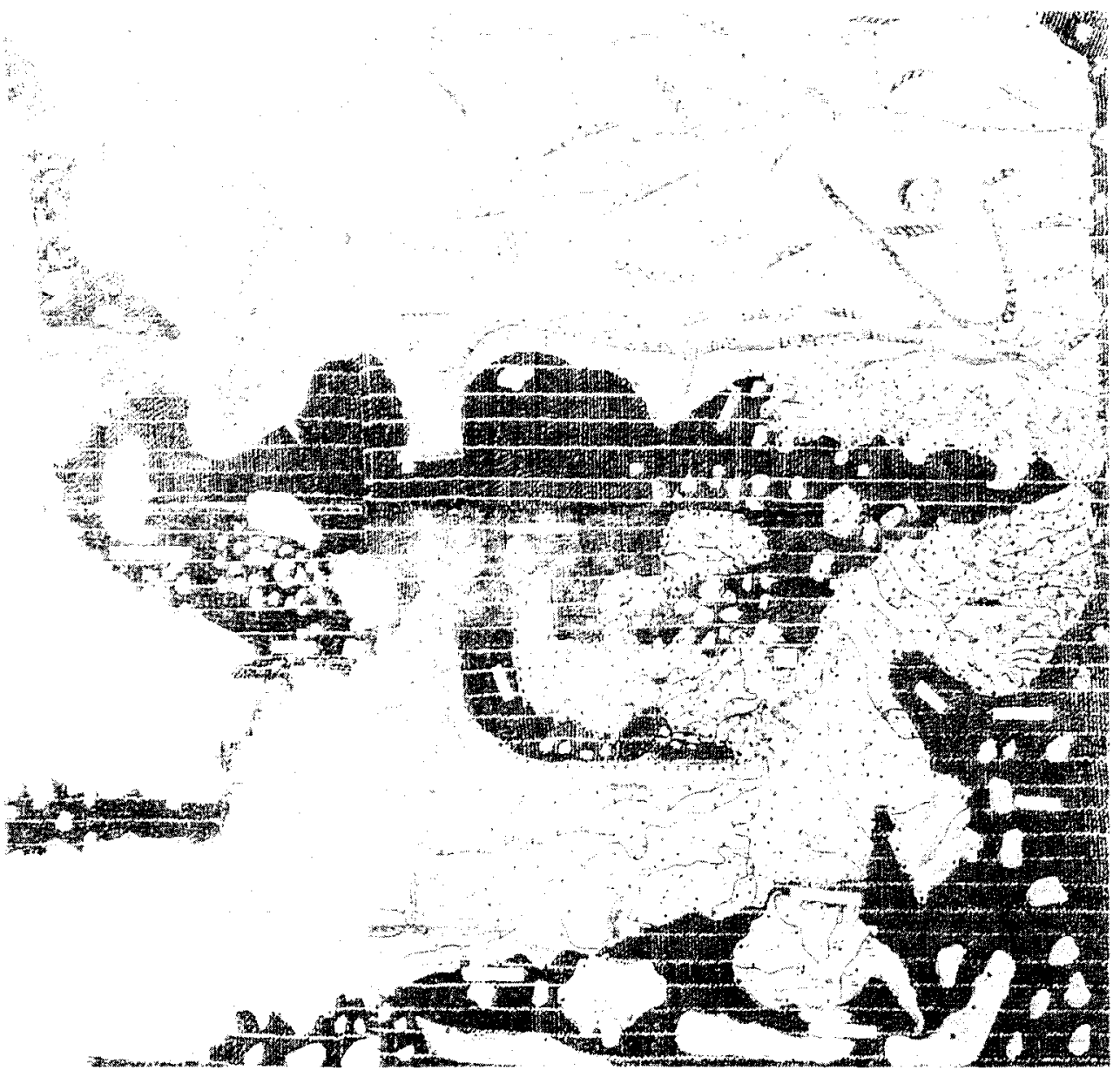

Fig. 4. Tabula Rogeriana de al Idrisi. Fuente: IGN. 1991. 


\section{BIBLIOGRAFIA}

Franco Aliaga. T. (2001) Viajar a través de la cartografia. Madrid: UNED.

FUNDACION SANTILLANA (1992) La imagen del mundo: 500 años de cartografia. Madrid: Fundación Santillana.

IBN KHALDUn (1967) The Muqaddymah: an introduction to history. Londres: Routedge an Kegan Paul.

instituto Geografico Nacional (1991) Atlas Nacional de España. Madrid: IGN.

Jaubert, P. A. (1975) La Géographie d'Édrisi. Amsterdan: Philo Press.

LACTANCIO, L. C. (1990) Instituciones divinas. Madrid: Gredos

Ortega VAlCARCEL, J. (2000) Los horizontes de la geografia. Barcelona: Ariel.

Unwin. T. (1995) El Lugar de la Geografia. Madrid: Cátedra. 